\title{
Valores del análisis citoquímico de líquido cefalorraquídeo en pacientes con derivación ventrículo-peritoneal
}

\author{
Marvid Sol Duarte Moreno', Germán Camacho-Moreno² y Nury Mancilla Gómez³
}

'Departamento de Pediatría Universidad Nacional de Colombia. Bogotá, Colombia ${ }^{2}$ Departamento de Pediatría Universidad Nacional de Colombia. Fundación Hospita de la Misericordia. Bogotá, Colombia

${ }^{3}$ Departamento de Pediatría

Universidad Nacional de Colombia, Bogotá, Colombia.

Recibido: 8 de octubre de 2018 Aceptado: 29 de marzo de 2019

Correspondencia a: Marvid Sol Duarte Moreno msduartem@gmail.com

Germán Camacho-Moreno gcamachom@unal.edu.co

Nury Mancilla Gómez nimancillag@unal.edu.co

\section{Values of cerebrospinal fluid cytochemistry in pediatric patients with ventriculoperitoneal shunt}

Background: The ventriculo-peritoneal shunt (VPS) is the treatment for hydrocephalus, the cerebrospinal fluid (CSF) is evaluated for the management of its complications; however, information on the values of the cytochemistry in this population is insufficient. Aim: To describe the characteristics of the CSF cytochemistry of children in VPS management. Methods: Descriptive observational study, developed in Bogotá (Colombia), from 2008 to 2016. VPS and related procedures records were reviewed. Patients between 6 months and 18 years were included. Results: A total of 285 records were reviewed, 31 samples were entered. The CSF values were, respectively, for the median and 90\% percentile: total leukocytes: 0 and 7 cells $/ \mathrm{mm}^{3}$, neutrophils: 0 and 6.8 cells/ $\mathrm{mm}^{3}$, lymphocytes: 0 and 2 cells $/ \mathrm{mm}^{3}$, proteins: 13.4 and $67.2 \mathrm{mg} / \mathrm{dL}$, glucose: 59 and $27.4 \mathrm{mg} / \mathrm{dL}$. Discussion: Glucose values evinced a normal rank towards the widest inferior limit with protein values exceeding the values expected. Cellularity is the variable with the lowest variation. Conclusions: The values of the CSF cytochemistry in patients with VPS are not comparable to those of the healthy population and should be interpreted according to the characteristics of this population.

Keywords: Cerebrospinal fluid; ependymitis; ventriculo-peritoneal shunt.

Palabras clave: Líquido cefalorraquídeo; ependimitis; derivación ventrículo-peritoneal.

\section{Introducción}

L a hidrocefalia, que corresponde al aumento de líquido en la cavidad craneal, tiene una prevalencia de 0,48 a 0,81 en 1.000 nacidos vivos ${ }^{1}$. Para su manejo, en muchas ocasiones es necesario la colocación de una DVP. Como referencia, en Estados Unidos de América son instaladas 33.000 derivaciones aproximadamente cada año².

Entre las complicaciones de las derivaciones ventriculares se encuentran: la disfunción de origen mecánico y las infecciones del sistema de derivación; esta primera representa $8-64 \%$ y la última, 3-29\% de todas las complicaciones $^{3-5}$.

Estas dos complicaciones tienen medidas terapéuticas totalmente diferentes, por lo que su diferenciación es vital en la práctica clínica. Sin embargo, una de las manifestaciones principales de las infecciones del sistema de derivación es la disfunción de éste, pudiendo presentarse como un síntoma aislado o asociado con otros síntomas sutiles, lo que dificulta el adecuado diagnóstico diferencial de estas entidades ${ }^{4,6}$.

Esto ha llevado a investigar cuáles valores de líquido cefalorraquídeo (LCR) son significativos en este grupo poblacional para inclinar la balanza hacia un lado u otro, lo que no ha sido una tarea fácil.
Como ya lo han descrito diferentes autores, se aprecian cambios de los valores en el LCR de esta población con respecto a los valores que se usan en población sana y que no se asocian a procesos infecciosos del sistema de derivación ${ }^{7-9}$

Los diferentes estudios se han enfocado en encontrar asociaciones entre síntomas clínicos y hallazgos de laboratorio que permitan identificar la presencia o no de infección del sistema de derivación, pero no se ha realizado un estudio con el objetivo específico de evaluar cuáles son los rangos de valores de LCR normal en este grupo poblacional ${ }^{7,8}$.

\section{Objetivo}

Describir las características bioquímicas y de celularidad del LCR de pacientes con hidrocefalia en manejo con derivación ventrículo-peritoneal en población pediátrica.

\section{Materiales y Métodos}

\section{Diseño del estudio}

Descriptivo, observacional, de serie de casos de temporalidad ambispectivo, desarrollado en la Fundación Hospital de la Misericordia en Bogotá (Colombia), entre los años 2008 y 2016. 
El estudio contó con la aprobación previa del Comité de Ética de la Fundación del Hospital de la Misericordia, en el acta $\mathrm{N}^{\circ}$ 02-02-10 del 12 de febrero de 2010. Según la Resolución No 008430 de 1993 (4 de octubre de 1993) en su artículo 11 este tipo de estudio corresponde a una investigación sin riesgo.

\section{Participantes del estudio}

Registros de niños entre 6 meses y 18 años fueron elegibles para inclusión si previamente venían usando un sistema de derivación ventricular y tenían una muestra tomada de LCR entre enero de 2008 y abril de 2016. Se obtuvo previamente consentimiento informado de aquelos pacientes recolectados de forma prospectiva. Para identificar los registros que cumplían estos criterios de inclusión se revisó la base de datos de los procedimientos de DVP y relacionados realizados en la institución. Los criterios de exclusión incluyeron la presencia de procesos febriles como motivo de consulta o previo al cambio del sistema de derivación, sospecha clínica de peritonitis, patología tumoral concomitante, cultivo de LCR y/o hemocultivo y/o cultivo del sistema de derivación ventricular positivo, uso de antimicrobianos en las dos semanas previas al cambio de sistema, neurocirugía previa en los últimos 14 días, necesidad de antibioterapia por sospecha de neuro-infección durante la hospitalización en la que se realizó el cambio del sistema de derivación y reingreso por infección del sistema de DVP o ventrículo-atrial en los siguientes tres meses tras la toma de la muestra de LCR. El objetivo de estos criterios de exclusión era eliminar aquellos pacientes que pudieran tener hallazgos anormales del LCR debidos a infección, cambios post-quirúrgicos o por patología tumoral en sistema nervioso central.

\section{Definiciones}

Fiebre: se definió como la presencia cuantificada de una temperatura axilar mayor a $38^{\circ}$. LCR traumático: se consideró como la presencia de más de 1.000 hematíes en la muestra, incluyendo, tanto eritrocitos frescos, como crenados. Se realizó corrección de proteínas y leucocitos según las fórmulas descritas en la literatura: $1,1 \mathrm{~g}$ de proteínas por 1.000 hematíes en $\mathrm{LCR}^{10}$ y CS$\mathrm{FWBC}=0,5 \times[\mathrm{CSFRBC} \times \mathrm{BloodWBC} / \mathrm{BloodRBC}]^{11}$, respectivamente. Proteína $C$ reactiva (PCR) elevada: aquella mayor al valor de referencia según el laboratorio (>6 mg/L). Leucocitosis: elevación de leucocitos totales superior a los valores de referencia esperados para la edad, según los valores manejados por el laboratorio institucional.

\section{Colección de datos y análisis estadísticos}

De todos los registros se recolectaron datos demográficos, clínicos, resultados de laboratorios de estudio de LCR y de estudios séricos, así como la evolución clínica a lo largo de los siguientes tres meses. Durante el periodo evaluado no hubo cambios en los procedimientos de laboratorio relacionados con los datos del estudio en la institución.

Los datos categóricos fueron descritos usando frecuencias y porcentajes y, dado que las variables continuas presentaron una distribución no normal, se describieron utilizando medianas, rangos intercuartílicos y percentiles. Para el análisis por subgrupos se utilizaron pruebas paramétricas y no paramétricas según el tipo de distribución de la variable evaluada. Los datos fueron analizados utilizando SPSS v25 (IBM SPSS Statistics).

\section{Resultados}

Se revisó un total de 285 registros; luego de aplicar los criterios de inclusión y exclusión se redujeron a 32 registros (Figura 1). Treinta y una muestras se tomaron del sistema ventricular: tres de ellas por punción transfontanelar y 28 en salas de cirugía durante el recambio del sistema de derivación; una de estas muestras se eliminó posteriormente ya que fue recolectada a través de punción lumbar. En total se incluyeron 31 muestras pertenecientes a un total de 25 pacientes, tomadas todas en el trascurso de diferentes hospitalizaciones. Tres de las muestras fueron recolectadas de forma prospectiva y 28 de forma retrospectiva.

En la Tabla 1 se describen las características de la población del presente estudio.

Los síntomas que llevaron a consultar a los pacientes fueron: cefalea (55\%), vómito (68\%), convulsiones (13\%) y dolor abdominal $(22 \%)$.

Con respecto a los estudios séricos, se identificó la presencia de leucocitosis en sangre periférica en 4/25 registros (16\%), y elevación de la PCR en 5/14 registros (36\%). Tres de estos últimos casos fueron de muestras tomadas en el periodo pre-quirúrgico, uno en el postquirúrgico, y el tiempo de la toma de la muestra anormal restante fue desconocido.

En la Tabla 2 se muestra la distribución de los datos obtenidos de leucocitos, proteínas y glucosa en LCR, incluyendo los datos corregidos y no corregidos. Del total de muestras, tres fueron consideradas como traumáticas, por lo que se corrigió su valor de proteínas; sólo en uno de estos casos fue necesario realizar corrección de leucocitos. Estas correcciones se realizaron según los valores descritos en Materiales y Métodos, sin evidenciarse diferencias significativas entre el percentil 0 al 90\%.

Con respecto a los valores de leucocitos totales, la mediana corresponde a 0 céls $/ \mathrm{mm}^{3}$, con un percentil $90 \%$ de 7 céls $/ \mathrm{mm}^{3}$; mientras para los neutrófilos la mediana es de 0 céls $/ \mathrm{mm}^{3}$, con un percentil $90 \%$ de 6,8 céls $/ \mathrm{mm}^{3} ; \mathrm{y}$, por último, para los linfocitos estos valores corresponden a 0 céls $/ \mathrm{mm}^{3}$ y 2 céls $/ \mathrm{mm}^{3}$ respectivamente. 
En relación con las proteínas, la mediana es de 13,4 $\mathrm{mg} / \mathrm{dL}$ y un percentil $90 \%$ de $67,2 \mathrm{mg} / \mathrm{dL}$.

Para la glucosa la mediana corresponde a $59 \mathrm{mg} / \mathrm{dL}$ y el percentil $10 \%$ de $27,4 \mathrm{mg} / \mathrm{dL}$.

Para evaluar la influencia que algunas variables podrían tener en los valores de LCR se realizaron diferentes análisis por subgrupos. Se evaluó las diferencias entre las muestras retrospectivas y prospectivas, así como según el antecedente de infección del sistema, sin encontrarse diferencias estadísticamente significativas (Material suplementario).

\begin{tabular}{|lll|}
\hline \multicolumn{2}{ll|}{ Tabla 1. Características de la población } \\
\hline Característica & & \\
\hline Sexo & Mujeres & $6 / 25(24 \%)$ \\
& Hombres & $19 / 25(76 \%)$ \\
\hline Edad & Mediana & 8,2 años \\
& Rango & $0,5-17,6$ años \\
\hline Procedencia & Bogotá y alrededores & $96 \%$ \\
& Costa Atlántica & $4 \%$ \\
\hline $\begin{array}{l}\text { Etiología de la } \\
\text { hidrocefalia }\end{array}$ & Obstructiva & $54 \%$ \\
\hline $\begin{array}{l}\text { Tiempo de uso } \\
\text { de la derivación }\end{array}$ & Mediana & $46 \%$ \\
\hline $\begin{array}{l}\text { Número de } \\
\text { recambios } \\
\text { previos }\end{array}$ & Rango & 95 meses \\
\hline $\begin{array}{l}\text { Infecciones } \\
\text { previas }\end{array}$ & $4-8$ & 3 meses-8,7 años \\
\hline $\begin{array}{l}\text { Tiempo desde } \\
\text { la infección }\end{array}$ & No & $0-8$ \\
\hline
\end{tabular}

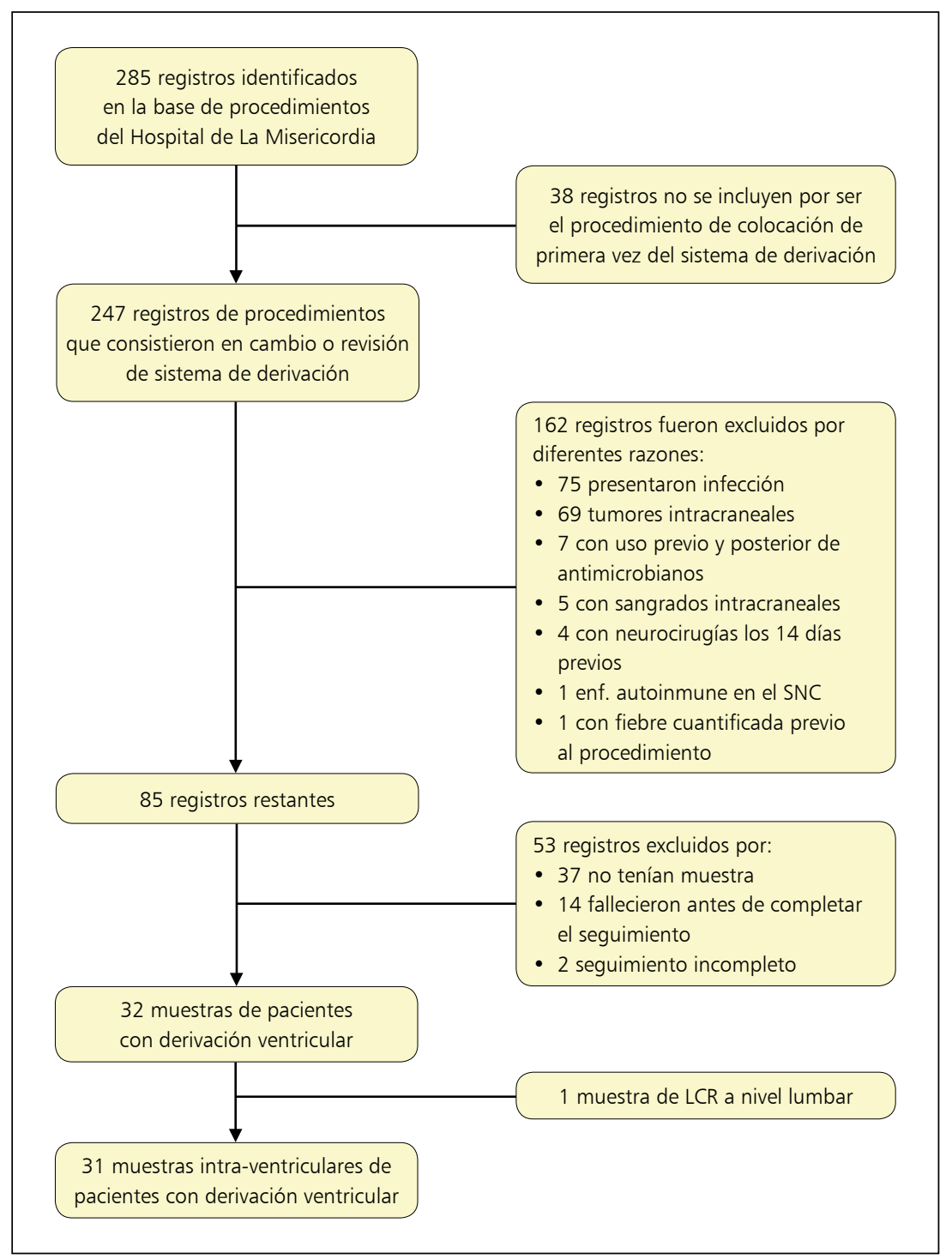

Figura 1. Flujograma de recolección de pacientes.

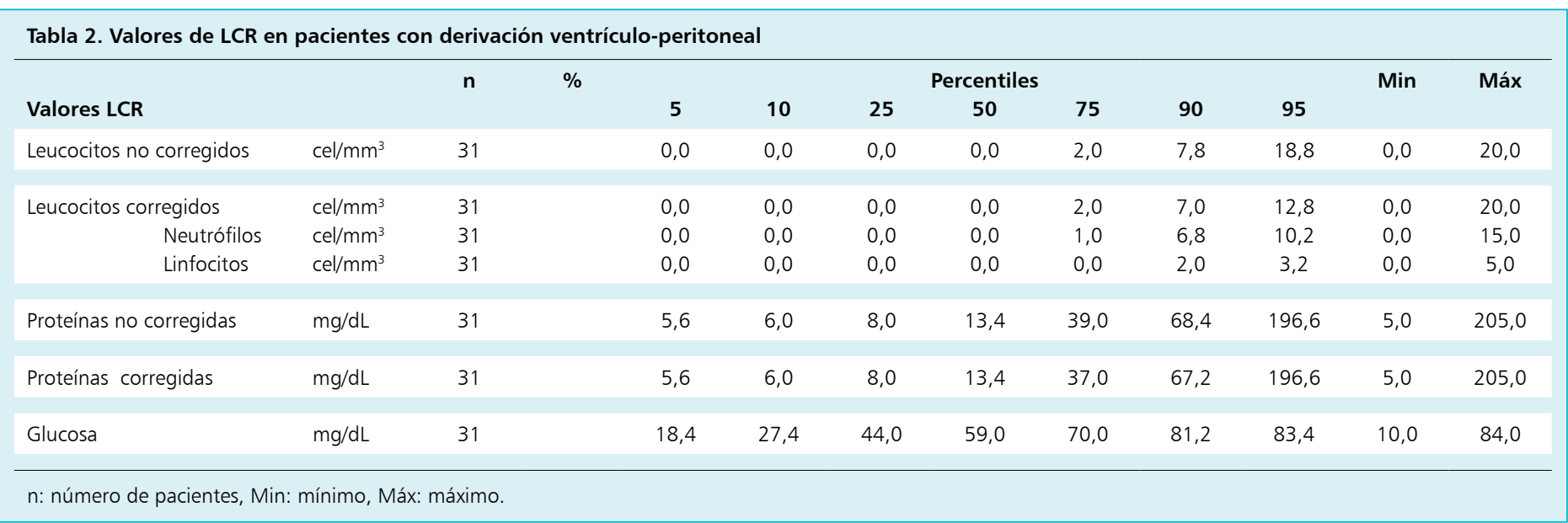


Con respecto al tiempo de derivación in situ $\left(\mathrm{T}_{\mathrm{dis}}\right)$, $81 \%$ de los pacientes había usado la derivación más de $90 \%$ de su vida y $90 \%$ más de $80 \%$ de su vida. Por lo anterior, los análisis por grupos etarios y por tiempo de derivación in situ arrojaron resultados similares (Material suplementario). Para estos dos análisis se agruparon los datos en cuatro grupos de la siguiente manera: de 0-4 años, de 5-8 años, 9-12 años y de más de 12 años. La única variable que mostró diferencias estadísticamente significativas en los dos casos fue la glucosa. La media de glucosa según el $\mathrm{T}_{\text {dis }}$ en el grupo de 0 a 4 años fue de $44 \mathrm{mg} / \mathrm{dL}$ y en el grupo de más de 12 años fue de $67,2 \mathrm{mg} / \mathrm{dL}$. La diferencia entre estos dos grupos fue de 23,26mg/dL, siendo esta diferencia estadísticamente significativa $(p=0,018)$. Hay una tendencia a ser mayor la glucosa en el grupo de 9 a 12 años (media de 65,3 $\mathrm{mg} / \mathrm{dL}$ ) en comparación con el grupo de 0-4 años, con una diferencia de $21,37 \mathrm{mg} / \mathrm{dl}$, sin llegar a ser estadísticamente significativa $(\mathrm{p}=0,067)$.

\section{Discusión}

Se examinaron los valores de leucocitos, glucosa y proteínas en el LCR de pacientes pediátricos, dado que, a pesar de ser un parámetro de amplio uso en la práctica clínica en este tipo de pacientes, desconocemos hasta el momento el rango de valores normales. Esta investigación es uno de los primeros estudios enfocado específicamente en establecer valores de normalidad específicos para este grupo de población, siendo estos hallazgos de gran importancia dado que el LCR es el parámetro de laboratorio más usado en la actualidad para ayudar a diagnosticar las infecciones del sistema de derivación ventricular y diferenciarlas específicamente de entidades patológicas de gran similitud clínica como lo es la disfunción mecánica del sistema de derivación.

En la literatura científica no hay consenso sobre que valores deben ser tomados como normales, y por lo tanto no hay claridad sobre qué valores son claramente patológicos; es así, que hay reportes de aproximaciones para definir infección de LCR con un recuento de leucocitos en LCR mayor de $100 / \mathrm{mm}^{3}$ [especificidad (E): $91 \%$ y valor predictor positivo (VPP): 0,46$]$ y neutrófilos mayores a $10 \%$ [sensibilidad (S): $82 \%$ y valor predictor negativo (VPN): 0,45$]^{7}$. Con respecto a los valores netos de leucocitos de LCR en pacientes con disfunción del sistema $(\mathrm{N}=69)$, refieren un valor de $49 \pm 169(0-1110)$ cél $1 / \mathrm{mm}^{3}$, con valores de neutrófilos de $7 \pm 18 \%(0-97 \%)$ y de linfocitos de $5 \pm 12 \%(0-49 \%)^{7}$; sin embargo, este estudio no tenía como objetivo principal evaluar valores normales de LCR en este grupo de pacientes, así que presenta mayor laxitud en los criterios de exclusión lo cual les permitió recolectar una muestra mayor pero no garantiza una muestra adecuada para este tipo de análisis disminuyendo su validez. Esto puede explicar las grandes diferencias en los valores de células obtenidos en este estudio a comparación del presente.

Con respecto al valor de los leucocitos usados en los estudios científicos para identificar infección del sistema ventricular, existe un margen importante de variabilidad, pudiendo ser anormal desde valores superiores a 5 céls/ $\mathrm{mm}^{3}$ hasta valores superiores a $25 \mathrm{céls} / \mathrm{mm}^{3}$, según el autor consultado $^{12-14}$. Se evidencia que todos manejan un rango diferente que se puede explicar por el poco conocimiento con el que se cuenta sobre este aspecto.

Con respecto a los valores normales de proteínas y glucosa de LCR, la literatura científica reporta para el primer caso valores menores de $45 \mathrm{mg} / \mathrm{dL}$ y en el segundo caso valores mayores de $45 \mathrm{mg} / \mathrm{dL}^{12-14}$. Siendo expuesto por otros autores que los pacientes con hidrocefalia hipertensiva pueden presentar hipoglucorraquia y aumento de la proteinorraquia no asociada a infección; sin embargo, dado la falta de valores normales, es variable el valor que se utilizan en la práctica clínica. En este estudio se muestra que los valores de glucosa presentan un rango normal hacia el extremo inferior más amplio que lo usado en pacientes sin derivación ventrículo-peritoneal, así como valores de proteínas que pueden ser mayores a los valores esperados en población normal.

No se encontró en los resultados que el cambio de hipoglucorraquia pueda deberse a un hallazgo residual de una infección previa del sistema de DVP. Para explicar este hallazgo se plantea como hipótesis una alteración del trasporte de la glucosa, secundario a un posible proceso de inflamación meníngeo, en este caso asociado a un cuerpo extraño en el SNC, siendo un mecanismo similar al propuesto en la neurosarcoidosis ${ }^{15,16}$.

Con respecto a las diferencias de glucosa encontradas en el análisis por grupos etarios y por tiempo de derivación in situ, y considerando a priori que estos valores no cambian por grupos de edad en pacientes sanos después de los 6 meses, es más probable que estas diferencias reflejen los cambios secundarios al tiempo de permanencia de la derivación in situ. Se considera como posible hipótesis para explicar este hallazgo una "normalización" del trasporte cerebral de glucosa que refleje una adaptación del SNC al uso crónico y prolongado de un sistema de derivación in situ. No se encontraron estudios al respecto para confrontar dicha hipótesis.

La elevación de la PCR en las muestras pre-quirúrgicas se consideró un hallazgo aislado inespecífico, lo cual ya ha sido descrito por otros autores ${ }^{17}$.

Entre las limitaciones de este estudio se encuentra el pequeño tamaño de la muestra; sin embargo, dado los estrictos criterios de exclusión aplicados recoger muestras amplias presenta bastante dificultad. Otra limitación es la presencia entre los registros de muestras 
traumáticas; sin embargo, es difícil no tener este tipo de muestras cuando a la mayoría de los pacientes se les tomó el examen a través de intervenciones neuroquirúrgicas. A pesar de esto la cantidad de muestras que se obtuvo catalogadas como traumáticas fueron bajas $(n=3)$ y se les aplicaron las medidas de corrección de proteínas y leucocitos descritos en la literatura médica para disminuir este sesgo.

También existe la limitación de que algunas muestras fueron tomadas a través de punción transfontanelar, mientras que la mayoría se recolectaron al finalizar la intervención quirúrgica dentro de las salas de cirugía, se desconoce si estas diferencias en la metodología pueden causar diferencias de resultados entre un grupo y otro; pero dado el limitado tamaño de la muestra no fue posible realizar un análisis separado según el método de recolección.

A pesar de las limitaciones descritas, consideramos que, dada la muy escasa información sobre este tópico, este estudio presenta una gran relevancia en la práctica clínica.

A futuro se deben realizar estudios con mayor tamaño de muestra para corroborar los hallazgos obtenidos, así como continuar en la búsqueda de valores que permitan diferenciar lo mejor posible entre disfunción mecánica e infección ventricular.

\section{Conclusiones}

Se demuestra que los valores de LCR en pacientes usuarios crónicos de derivación ventrículo-peritoneal son diferentes a los valores clásicamente descritos en población sana, por lo cual la extrapolación de estos últimos a los primeros puede llevar a errores diagnósticos y de tratamiento. De los ítems evaluados, los valores que presentan un rango más estrecho y son más confiables en la práctica clínica es la celularidad. Los valores de glicemia que se pueden obtener son más bajos de lo esperado para pacientes sanos. Por último, las proteínas presentan un rango muy amplio entre sus valores lo que lleva a que sean de menor utilidad para la práctica clínica diaria.

\section{Resumen}

Introducción: La derivación ventrículo-peritoneal (DVP) es el tratamiento para la hidrocefalia. El líquido cefalorraquídeo (LCR) se evalúa para el manejo de sus complicaciones; sin embargo, la información de los valores del citoquímico en esta población es insuficiente. Objetivo: Describir las características del citoquímico del LCR de niños en manejo con DVP. Materiales $y$ Métodos: Estudio de tipo observacional descriptivo, desarrollado en Bogotá (Colombia), entre el año 2008 y 2016. Se revisaron los registros de procedimientos de DVP y relacionados. Se incluyeron pacientes entre 6 meses y 18 años de edad. Resultados: Se revisaron 285 registros e ingresaron 31 muestras. Los valores de LCR fueron, respectivamente, para la mediana y al percentil 90\%: leucocitos totales: 0 y 7 céls $/ \mathrm{mm}^{3}$, neutrófilos: 0 y 6,8 céls $/ \mathrm{mm}^{3}$, linfocitos: 0 y 2 céls $/ \mathrm{mm}^{3}$, proteínas: 13,4 y 67,2 mg/dL, glucosa: 59 y 27,4 mg/dL. Discusión: Los valores de glucosa presentan un rango normal hacia el extremo inferior más amplio, con valores de proteínas mayores a los valores esperados. El rango de celularidad es la variable que presenta menor variación. Conclusiones: Los valores del citoquímico de LCR en paciente con DVP no son equiparables a los de la población sana y deben interpretarse según las características propias de esta población.

\section{Referencias bibliográficas}

1.- Chumas P, Tyagi A, Livingston J. Hydrocephalus-what's new? Arch Dis Child Fetal and Neonatal Ed. 2001; 85 (3): F149-F154. doi: 10.1136/fn.85.3.F149.

2.- Bondurant CP, Jimenez DF. Epidemiology of cerebrospinal fluid shunting. Pediatr Neurosurg 1995; 23(5): 254-8; discussion 259. doi: 10.1159/000120968.

3.- Díaz Padilla C, López Vásquez G A, Pérez Ramírez J D, Palacios Saucedo G. Hidrocefalia, derivación ventricular y ependimitis (Parte I). Enf Infec Microbiol 2016; 23 (2): 38-43. http:// www.medigraphic.com/pdfs/micro/ei-2003/ ei032e.pdf.

4.- Díaz Padilla C, López Vásquez G A, Pérez Ramírez J D, Palacios Saucedo G. Hidrocefalia, derivación ventricular y ependimitis (Parte II). Enf Infec Microbiol 2016; 23 (2): 44-9. http:// www.medigraphic.com/pdfs/micro/ei-2003/ ei032f.pdf.

5.- Wong J M, Ziewacz J E, Ho A L, Panchmatia JR, Bader AM, Garton HJ, et al. Patterns in neurosurgical adverse events: cerebrospinal fluid shunt surgery. Neurosurg Focus. 2012; 33(5): E13. doi: 10.3171/2012.7.FOCUS12179.

6.- García-Vázquez E, Gómez J. Tratamiento de las infecciones relacionadas con procedimientos neuroquirúrgicos. Rev Española Quimioter 2007; 20 (1): 36-43. PMID: 17530034.

7.- McClinton D, Carraccio C, Englander R. Predictors of ventriculoperitoneal shunt pathology. Pediatr Infect Dis J. 2001; 20 (6): 593-7. PMID: 11419501.

8.- Lan C C, Wong T T, Chen S J, Liang M L, Tang
R B. Early diagnosis of ventriculoperitoneal shunt infections and malfunctions in children with hydrocephalus. J Microbiol Immunol Infect 2003; 36 (1): 47-50. PMID: 12741733.

9.- Odio C M, CCSS SJ, Costa Rica, Huertas E, CCSS SJ, Costa Rica. Infecciones del líquido cefalorraquídeo en pacientes con derivaciones ventrículo peritoneales. Acta Pediatr Costarric. 2001; 15(1): 16-23. http://www.scielo. sa.cr/scielo.php?script=sci_arttext\&pid $=$ S1409-00902001000100003.

10.- Nigrovic LE, Shah S S, Neuman M I. Correction of cerebrospinal fluid protein for the presence of red blood cells in children with a traumatic lumbar puncture. J Pediatr 2011; 159 (1): 158-9. doi: 10.1016/j.jpeds.2011.02.038.

11.- Rizvi S, Moein-Afshari F. Redefining true leukocytosis in the traumatic lumbar 
puncture. Can J Neurological Sci / J Can Sci Neurol. 2015; 42(Supplement S1): S30-S30. doi:10.1017/cjn.2015.142.

12.- Prusseit J, Simon M, von der Brelie C, et al. Epidemiology, prevention and management of ventriculoperitoneal shunt infections in children. Pediatr Neurosurg 2009; 45 (5): 325 36. doi: $10.1159 / 000257520$

13.- Wang K W, Chang W N, Shih T Y, Huang C R, Tsai N W, Chang C S, et al. Infection of cerebrospinal fluid shunts: causative pathogens, clinical features, and outcomes. Jpn J Infect Dis 2004; 57 (2): 44-8. PMID: 15118207.

14.- von der Brelie C, Simon A, Groner A, Molitor E, Simon M. Evaluation of an institutional guideline for the treatment of cerebrospinal fluid shunt-associated infections. Acta Neurochir (Wien). 2012; 154(9): 1691-7. doi: 10.1007/s00701-012-1329-x

15.- Sarva H, Chapman R, Omoregie E, Abrams C. The challenge of profound hypoglycorrhachia: two cases of sarcoidosis and review of the literature. Clin Rheumatol 2011; 30: 1631-9. doi: 10.1007/s10067-0111834-y.

16.- Viola G. Extreme hypoglycorrhachia: Not always bacterial meningitis. Nat Rev Neurol 2010; 6: 637-41. doi: 10.1038/ nrneurol.2010.126.

17.- Lolak S, Bunyaratavej K. C-reactive protein in prediction of ventriculoperitoneal shunt-related infection in high-risk patients. Surg Infect 2013; 14 (2): 192-5. doi: 10.1089/sur.2011.070. 